\title{
MEMS FILTER WITH VOLTAGE TUNABLE CENTER FREQUENCY AND BANDWIDTH
}

\author{
Lih Feng Cheow, Hengky Chandrahalim, and Sunil A. Bhave \\ OxideMEMS Lab, 102 Phillips Hall, Cornell University, Ithaca, NY 14853, USA
}

\begin{abstract}
This paper reports on the design of a reconfigurable ladder filter using RF MEMS resonators with voltage-tunable series and parallel resonance frequencies. The ladder filter consists of one shunt and two series resonators operating in the half-wave thickness shear vibration mode. It demonstrates a center frequency tuning range of $8 \mathrm{MHz}$ at $817 \mathrm{MHz}$ and an adjustable bandwidth from $600 \mathrm{kHz}$ to $2.8 \mathrm{MHz}$, while maintaining an insertion loss $<4$ $\mathrm{dB}$, stop-band rejection $>30 \mathrm{~dB}$ and pass-band ripple $<2 \mathrm{~dB}$. This voltage tunable design enables channel agility and reconfigurability, substantially reducing the filter count in channelselect radio receiver architectures. Finally, a simple algorithm is provided to facilitate dynamic tuning of filter center frequency and bandwidth.
\end{abstract}

\section{INTRODUCTION}

Multi-band, multi-standard radio receivers require a large array of channel-select filters connected in parallel. The input capacitance of the filter array will 'load' individual filters, deteriorating their stop-band rejection. For reconfigurable radios the front-end filters must also handle encoded waveforms with different bandwidth requirements. A filter with dynamically tunable center frequency and bandwidth will not only overcome fabrication tolerances and thermal drift, but will also reduce capacitive loading at the filter input, enable handling of multiple waveforms, and substantially decrease the number of filters in next-generation receivers.

Low frequency filters comprised of electrostatically-coupled resonators have been demonstrated with $10 \times$ bandwidth tunability [1]. However, it is challenging to implement electrostatic coupling springs at $\mathrm{GHz}$ frequencies even with $100 \mathrm{~nm}$ air-gaps. Galayko et al presented a tunable bandwidth filter using clamped-clamped beam resonators in a ladder configuration [2]. The first transmission zero (and hence filter bandwidth) was tuned by controlling the series resonance frequency of the shunt resonator, though large parasitic capacitance prevented implementation and tunability of the second transmission zero of the filter.

In a typical ladder filter configuration, $\omega_{\text {parallel }}$ of the shunt resonator, which defines the filter center frequency $f_{c}$, is matched to $\omega_{\text {series }}$ of the series resonators. Filter bandwidth (BW) is determined by notches on either side of the pass-band and is $2 \times$ the pole-zero separation of the series and shunt resonators. The key to tunable ladder filters is the ability to change $f_{c}$ and to dynamically tune the pole-zero separation $\omega_{\text {parallel }}-\omega_{\text {series }}$ of the resonators. We have previously demonstrated channel-select ladder filters with $600 \mathrm{kHz}$ bandwidth, $25 \mathrm{~dB}$ stop-band rejection, excellent shape factor, and low insertion loss (IL) using dielectrically-transduced thickness shear mode resonators [3]. In this paper, we introduce a voltage biasing scheme capable of independently tuning the series resonance and pole-zero separation of the filter's constituent resonators. Coupled with orthogonal frequency tuning [4], we can configure the filter with desired pass-band characteristic in real time.

\section{ORTHOGONAL FREQUENCY TUNING}

The series resonance of low frequency resonators can be tuned by electrostatic spring tuning. However, the stiffness of high frequency resonators is quite large (a $1 \mathrm{GHz}$ bulk-mode resonator has stiffness on the order of $1 \mathrm{MN} / \mathrm{m}$ in the resonant direction) and would require considerable electrostatic force to tune the stiffness and the series resonance frequency.

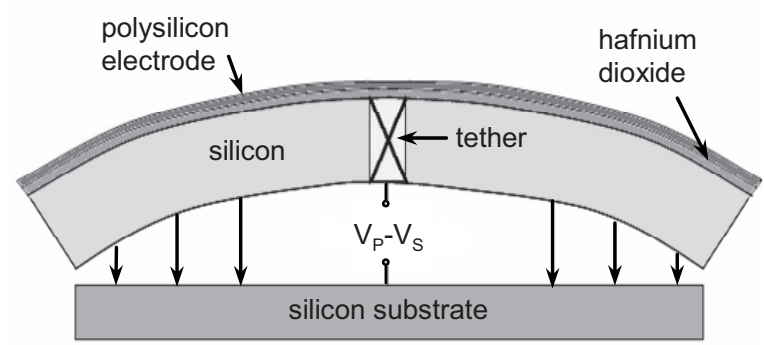

Figure 1. Longitudinal cross-section of a half-wave thickness shear mode resonator. The tuning voltage $V_{p}-V_{s}$ generates a vertical force and bends the silicon bar in the vertical direction, thereby changing the effective stiffness of the resonator.

In contrast, orthogonal frequency tuning deforms the resonator in a direction perpendicular to the direction of vibration. The resonators are generally less stiff in the orthogonal direction and can be deformed with substantially less force. The symmetric half-wave thickness shear mode resonator exhibits a small-amplitude flexure mode coupled to the shear mode [3]. The Southwell-Dunkerley formula approximates the combined shear-flexure frequency as $\frac{1}{f_{\text {total }}^{2}}=\frac{1}{f_{\text {shear }}^{2}}+\frac{1}{f_{\text {flexure }}^{2}}$.

When a polarization voltage $V_{p}$ is applied to the resonator and a voltage $V_{s}$ is applied to the substrate, the tuning voltage $V_{p}-V_{s}$ generates an electrostatic force that deflects the structure towards the substrate, as illustrated in Figure 1. Bending the structure softens the flexural mode stiffness, lowering the series resonance frequency.

\section{POLE-ZERO SEPARATION TUNING}

A dielectrically transduced MEMS resonator can be represented by an equivalent series LCR circuit in parallel with a feedthrough capacitance $C_{f t}$, as shown in Figure 2. For a given transduction efficiency $\eta \equiv V_{p} \partial C / \partial x, R_{X}=b / \eta^{2}, C_{X}=\eta^{2} / K$, and $L_{X}=M / \eta^{2}$, where $b, K$ and $M$ denote the damping constant, effective spring stiffness and effective mass of the resonator. The feedthrough capacitance in a two-port resonator originates from electric field coupling from the input electrode to the output electrode and is a function of electrode geometry. The series resonance frequency is given by

$\omega_{\text {series }}=\frac{1}{\sqrt{L_{x} C_{x}}}=\sqrt{\frac{K}{M}}$. 


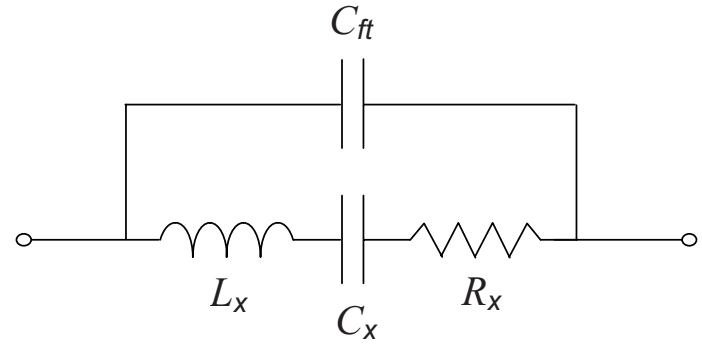

Figure 2. Equivalent circuit of a dielectrically transduced MEMS resonator.

An expression for the parallel resonance frequency is obtained through a first-order Taylor's expansion

$$
\begin{aligned}
& \omega_{\text {parallel }}=\frac{1}{\sqrt{L_{x} \frac{C_{x} C_{f t}}{C_{x}+C_{f t}}}}=\omega_{\text {series }} \sqrt{1+\frac{C_{x}}{C_{f t}}} \approx \omega_{\text {series }}\left(1+\frac{C_{x}}{2 C_{f t}}\right) \\
& \omega_{\text {parallel }}-\omega_{\text {series }} \approx \omega_{\text {series }} \frac{C_{x}}{2 C_{f t}} \\
& \frac{\Delta\left(\omega_{\text {parallel }}-\omega_{\text {series }}\right)}{\Delta \omega_{\text {series }}} \approx \frac{C_{x}}{2 C_{f t}} .
\end{aligned}
$$

For electrostatic transduction, the ratio of $C_{X}$ to $C_{f t}$ is very small $\left(10^{-4}-10^{-2}\right)$. Therefore, the separation between the series and parallel resonances is largely independent of the series resonance frequency shifts due to changes in the spring constant $K$. The polezero separation can be modeled as a function of structure bias voltage $V_{p}$

$$
\omega_{\text {parallel }}-\omega_{\text {series }}=\left(\frac{\varepsilon^{2} A^{2}}{2 d^{4} C_{f t} \sqrt{K M}}\right) V_{p}^{2} \text {. }
$$

In other words, the parallel resonance frequency is simply a voltage-controlled offset from the series resonance frequency.

\section{FILTER TUNING ALGORITHM}

A ladder filter consists of a shunt resonator and two series resonators. For minimum insertion loss and pass-band ripple, the parallel resonance frequency of the shunt resonator is matched to the series resonance frequency of the series resonators. Ladder filters can be cascaded to provide higher stop-band rejection at the expense of insertion loss. To achieve the desired center frequency and bandwidth, we use the following filter synthesis method:

1. Fix $V_{p}$ and change $V_{s}$ for the series and shunt resonators such that the desired series resonance frequencies are obtained (orthogonal frequency tuning).

2. Tune $V_{p}-V_{s}$ separately for each resonator to obtain the desired pole-zero offset. Since $V_{p}-V_{s}$ remains constant, the bending of the resonators does not change so the series resonance frequency remains fixed.

\section{FABRICATION PROCESS}

Filter T-sections consisting of one shunt and two series resonators shown in Figure 3 were fabricated on an SOI wafer with a $3 \mu \mathrm{m}$ heavily doped device layer and $0.5 \mu \mathrm{m}$ buried oxide. The resonators are $310 \mu \mathrm{m}($ and $300 \mu \mathrm{m}) \times 100 \mu \mathrm{m} \times 3.1 \mu \mathrm{m}$ released

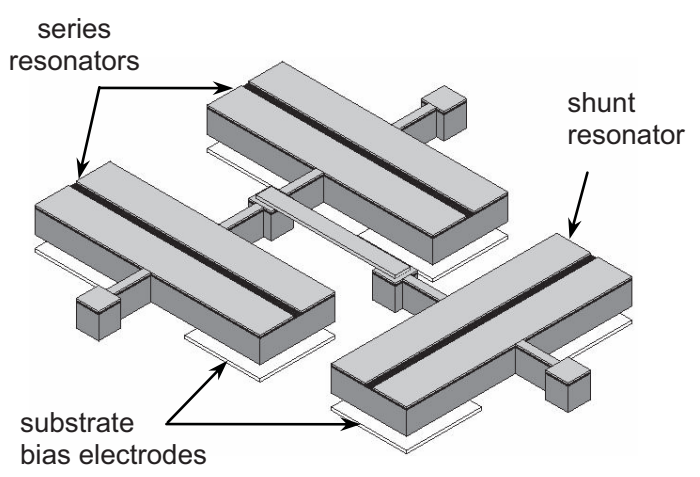

Figure 3. 3D model of tunable ladder filter.

silicon bars with $20 \mathrm{~nm}$ of hafnium dioxide and $50 \mathrm{~nm}$ of polysilicon layers on top for dielectric transduction. Orthogonal frequency tuning was achieved by applying a substrate bias voltage to bend the resonators in the vertical direction. A back-side etch was added to the fabrication process in [3] to create substrate islands for independent tuning of the resonators. A front-side trench etch allowed isolated DC bias voltages to be applied to the resonators (Figure 4). Process limitations prevented polysilicon connections between the series and shunt resonators, so a small gold bondwire was implemented as seen in Figure 5.

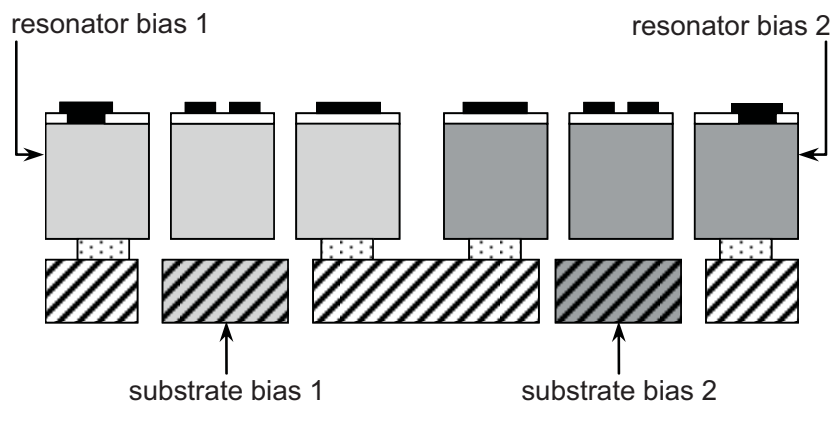

Figure 4. Device cross-section with isolated resonator and substrate for applying independent tuning voltages.

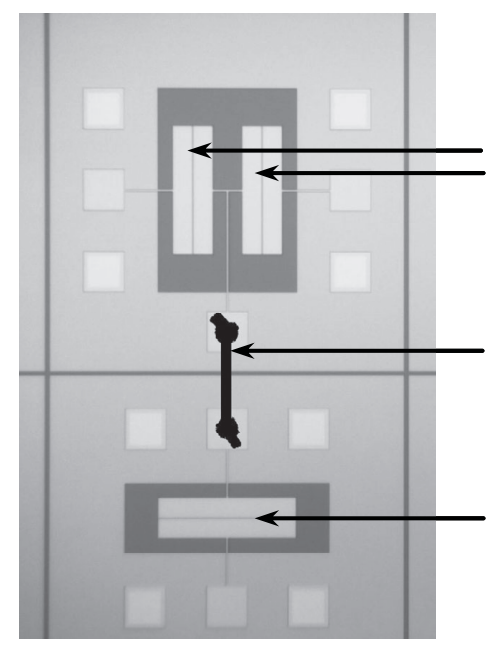

series

resonators

gold

wirebond

shunt

resonators

$300 \mu \mathrm{m}$

Figure 5. Microphotograph of tunable ladder filter with a gold wirebond bridge. Care must be taken to ensure that the wirebond does not damage the oxide under the pads and short the devices. 


\section{EXPERIMENTAL RESULTS}

The resonators and filters were characterized using a DesertCryo microwave probe station. The resonator proof-mass was grounded and a DC bias $V_{p}$ was applied to both the drive and sense electrodes with MiniCircuits bias-Ts. Quality factor characterization and $\mathrm{S}_{21}$ transmission measurements were performed using an Agilent 8722ES Network Analyzer. The resonators and filters were terminated with $50 \Omega$ and $500 \Omega$ impedances, respectively.

Measurement results demonstrating orthogonal frequency tuning are shown in Figure 6 . Keeping $V_{p}$ constant at $5 \mathrm{~V}$ and varying the substrate bias $V_{s}$ from $5 \mathrm{~V}$ to $17 \mathrm{~V}$, we can tune the series resonance frequency of a single resonator from $816 \mathrm{MHz}$ to $802 \mathrm{MHz}$, while maintaining a quality factor $Q>7000$. The parallel and series resonance separation does not change during orthogonal frequency tuning.

Figure 7 shows that the pole-zero separation of the resonator varies from $0.6 \mathrm{MHz}$ to $1.6 \mathrm{MHz}$ when $V_{p}$ is changed from $5 \mathrm{~V}$ to $12 \mathrm{~V}$. The substrate bias $V_{s}$ was held at $V_{p}$ during this measurement to prevent orthogonal forces acting on the resonator.

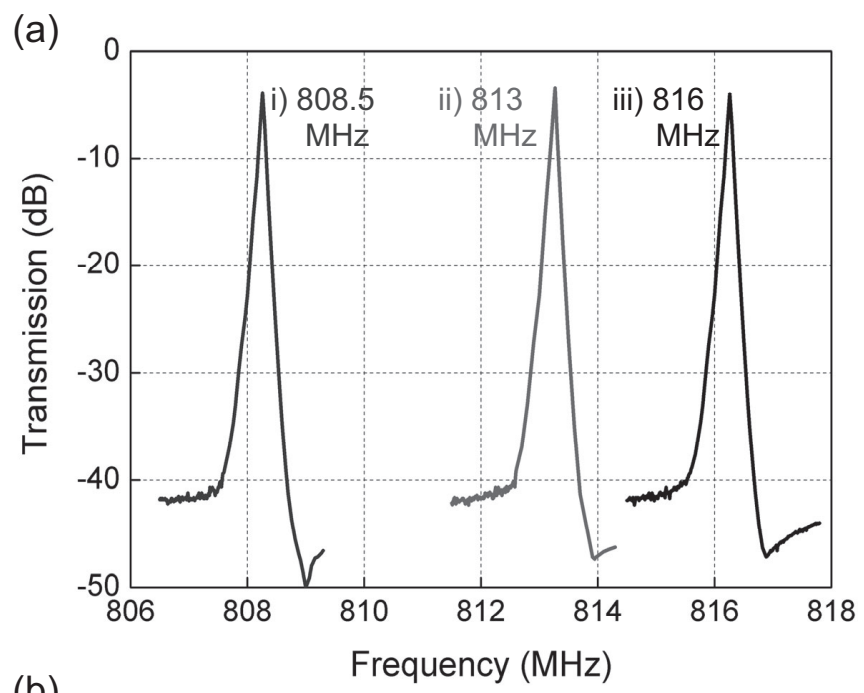

(b)

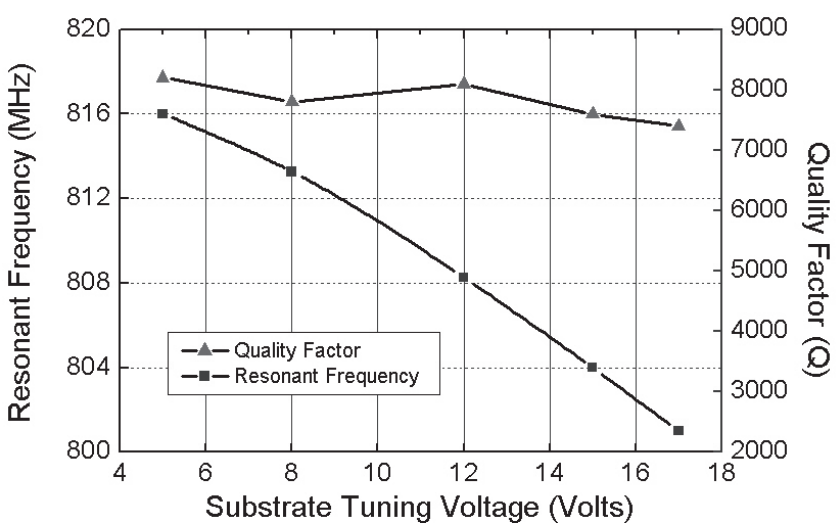

Figure 6. (a) Measured series resonance tuning for $V_{s}=5 \mathrm{~V}$ and $V_{s}$ = (i) $12 \mathrm{~V}$, (ii) $8 \mathrm{~V}$, and (iii) $5 \mathrm{~V}$. Orthogonal frequency tuning shifts series and parallel resonances by equal amount. (b) Resonant frequency tuning vs. substrate voltage. A tuning range of $15 \mathrm{MHz}$ is observed while maintaining $Q>7000$. (a)

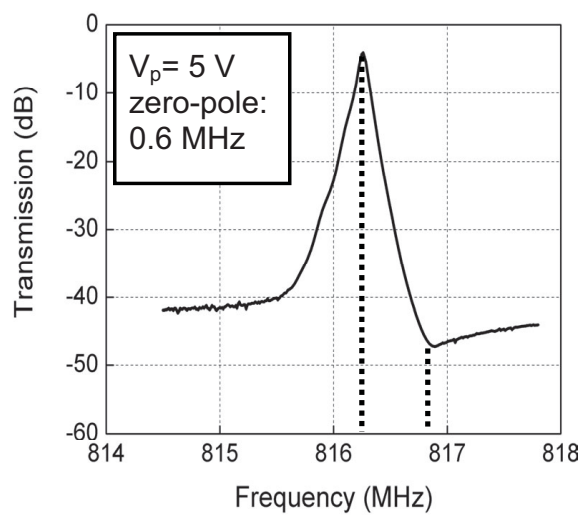

(b)

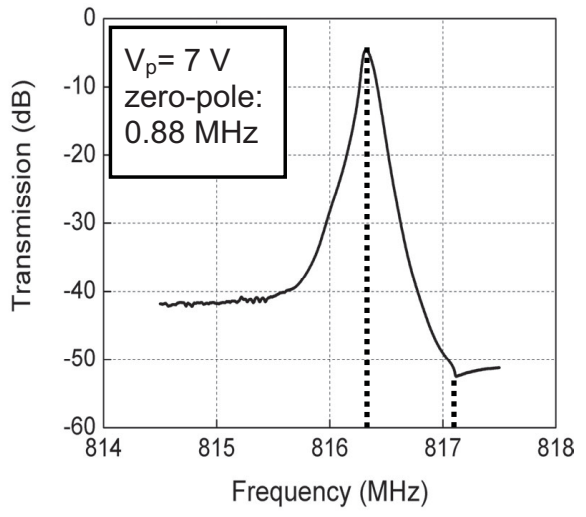

(c)
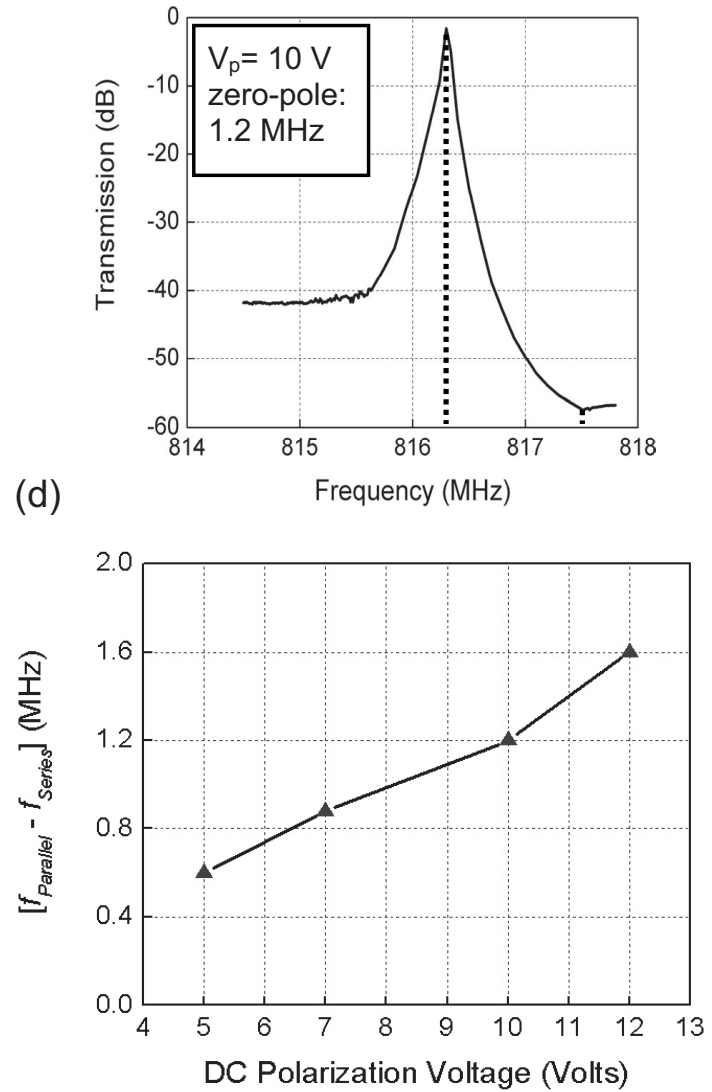

Figure 7. $(a, b, c)$ Measured transmission response demonstrating pole-zero separation of a thickness shear mode resonator as DC bias $V_{p}$ increases from $5 \mathrm{~V}$ to $10 \mathrm{~V}$. (d) Measured pole-zero separation vs. DC bias voltage of the resonator. 
A bias voltage $V_{p}=5 \mathrm{~V}$ yields a pass-band with $f_{c}=817.2 \mathrm{MHz}$, $0.6 \mathrm{MHz}$ bandwidth, and IL of $3.2 \mathrm{~dB}$ (Figure 8). By applying $V_{s}=$ $12 \mathrm{~V}$ and $15 \mathrm{~V}$ to the shunt and series resonators respectively, the center frequency is tuned from $817 \mathrm{MHz}$ to $809 \mathrm{MHz}$ without degradation in IL (3.5 dB) and shape factor (1.3), as shown in Figure 9.

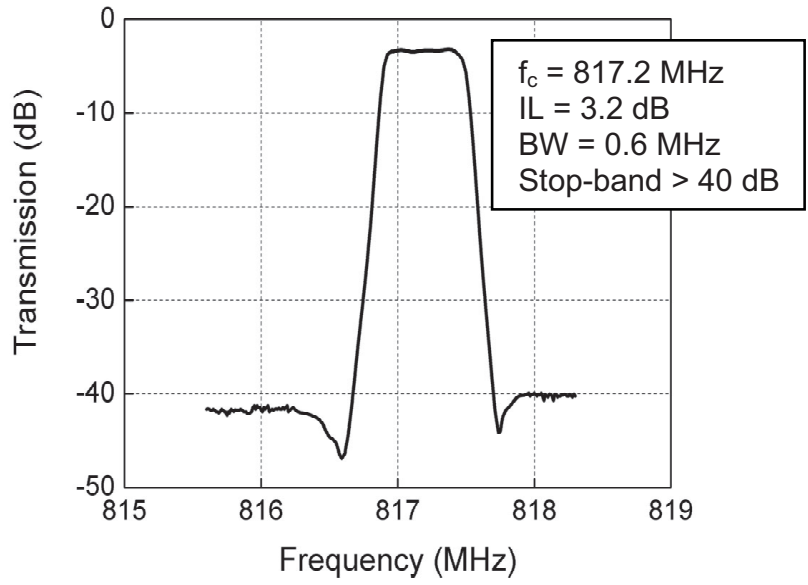

Figure 8. Measured transfer function of ladder filter with no tuning. Resonator and substrate bias voltages are at $5 \mathrm{~V}$.

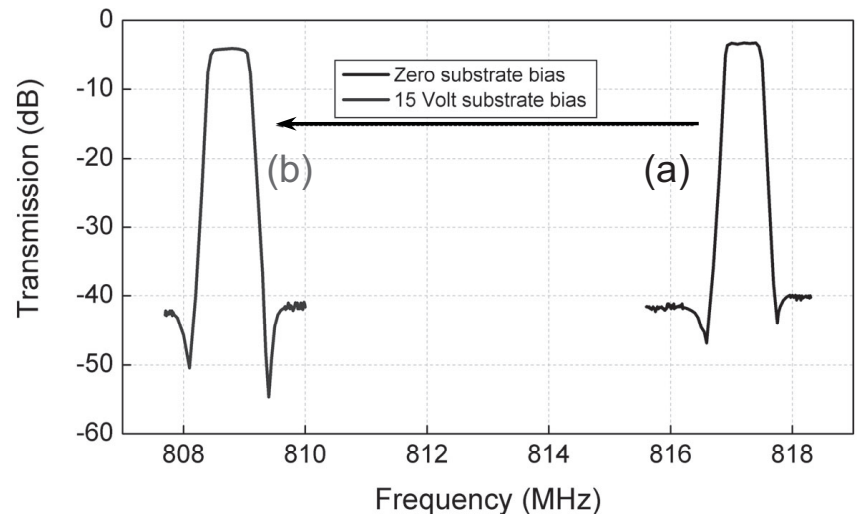

Figure 9. Measured transmission of ladder filter with center frequency tuning from $817 \mathrm{MHz}$ to $809 \mathrm{MHz}$ with (a) no tuning, and (b) series resonator: $V_{p}=5 \mathrm{~V}, V_{s}=15 \mathrm{~V}$; shunt resonator: $V_{p}=5 \mathrm{~V}$, $V_{s}=12 \mathrm{~V}$.

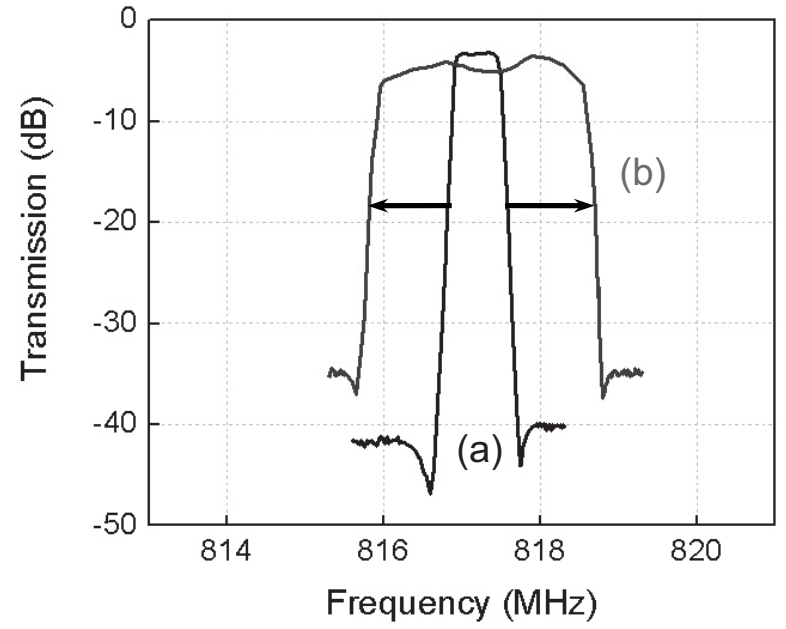

Figure 10. Measured transmission of ladder filter with bandwidth tuning from $0.6 \mathrm{MHz}$ to $2.8 \mathrm{MHz}$ with (a) no tuning, (b) series resonator: $V_{p}=12 \mathrm{~V}, V_{s}=12 \mathrm{~V}$; shunt resonator: $V_{p}=13 \mathrm{~V}, V_{s}=16 \mathrm{~V}$.

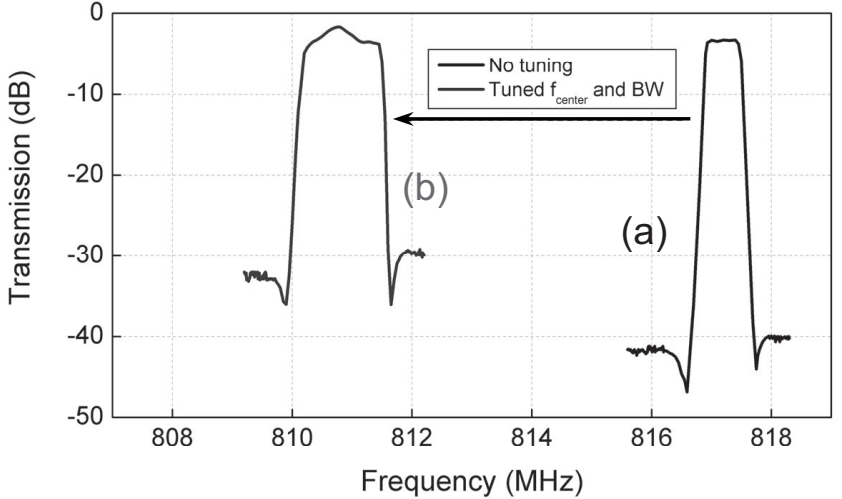

Figure 11. Measured transfer function of ladder filter with bandwidth tuning from $0.6 \mathrm{MHz}$ to $1.4 \mathrm{MHz}$ and center frequency tuning from $817 \mathrm{MHz}$ to $811 \mathrm{MHz}$ with (a) no tuning, and (b) series resonator: $V_{p}=10 \mathrm{~V}, V_{s}=19 \mathrm{~V}$; shunt resonator: $V_{p}=9 \mathrm{~V}$, $V_{s}=17 \mathrm{~V}$.

Figure 10 shows the bandwidth tuning from $0.6 \mathrm{MHz}$ to $2.8 \mathrm{MHz}$ while maintaining a constant center frequency at $817.2 \mathrm{MHz}$. However, the pass-band ripple increased from $0.4 \mathrm{~dB}$ to $1.8 \mathrm{~dB}$. Finally, a combination of bandwidth and center frequency tuning is shown in Figure 11. A pass-band with $f_{c}=811 \mathrm{MHz}$ and $1.4 \mathrm{MHz}$ bandwidth is obtained.

\section{CONCLUSIONS}

Voltage tunability is more versatile than one-time modifications like laser trimming and mass loading, and enables us to overcome process tolerance and temperature variation frequency shifts. We have demonstrated bandwidth and center frequency tunability in an RF MEMS filter with IL $<4 \mathrm{~dB}$ and stop-band rejection $>30 \mathrm{~dB}$. With process tolerance and temperature variation frequency shifts of $\pm 1.2 \mathrm{MHz}$ and $-14 \mathrm{ppm} /{ }^{\circ} \mathrm{C}$, respectively, this tuning capability not only overcomes these variations, but also enables channel agility and adaptability in multi-mode radio receivers.

\section{ACKNOWLEDGEMENTS}

The authors wish to thank the Cornell Center for Nanoscale Systems and RF Micro Devices, whose generous grant made this research possible, and the Cornell Nanofabrication Facility for device fabrication.

\section{REFERENCES}

[1] S. Pourkamali et al, "Electrostatically coupled micromechanical beam filters," MEMS 2004, Maastricht, The Netherlands, January 25-29, 2004, pp. 584-587.

[2] D. Galayko et al, "Tunable passband T-filter with electrostatically-driven polysilicon micromechanical resonators," Sensors and Actuators A: Physical, vol. 117, issue 1, January 3, 2005, pp.115-120.

[3] H. Chandrahalim et al, "Channel-select micromechanical filters using high-K dielectrically transduced MEMS resonators," MEMS 2006, Istanbul, Turkey, January 22-26, 2006, pp. 894-897.

[4] H. Chandrahalim et al, "Thickness shear mode vibrations in silicon bar resonators," Ultrasonics 2005, Rotterdam, The Netherlands, September 18-21, 2005, pp. 898-901. 